\title{
Thermal imaging application in chronic venous disease
}

\author{
Pilot study
}

\author{
Armand Cholewka · Agata Stanek • \\ Aleksandra Klimas • Aleksander Sieroń • \\ Zofia Drzazga
}

Received: 6 July 2013/Accepted: 2 August 2013/Published online: 1 September 2013

(c) The Author(s) 2013. This article is published with open access at Springerlink.com

\begin{abstract}
Thermal imaging was used for the estimation of lower-limb primary chronic venous diseases connected with an insufficiency of superficial veins. The current study was performed by means of a Thermovision A40 camera. The curves of the chosen regions of interest were performed in a research room with a stabilized temperature. Curves of the lower extremities showed that the patients suffered from a venous insufficiency characterized by a higher skin temperature connected with pathological changes in the veins. Changes seen in the lower extremities skin thermal map may be associated with blood stasis, inflammatory states and swelling. The differences in temperature distribution are due to a correlation between the medical diagnosis and the parameters obtained from duplex scanning. The results of the thermal imaging showed differences between patients' health may suggest that a thermovision of lower extremities venous diseases can be very useful in medical diagnosis. The correlation was obtained from temperature parameters and duplex scanning. The parameters may suggest that thermovision diagnostics may be useful as a complementary method in the diagnosis of chronic venous diseases in the lower extremities.
\end{abstract}

Keywords Thermovision diagnostics · Temperature · Primary chronic venous disease · Duplex scanning

A. Cholewka $(\bowtie) \cdot$ A. Klimas $\cdot$ Z. Drzazga

A. Chełkowski Institute of Physics, Department of Medical

Physics, University of Silesia, Uniwersytecka 4,

40-007 Katowice, Poland

e-mail: armand.cholewka@gmail.com

A. Stanek · A. Sieroń

Chair and Clinic of Internal Diseases and Physical Medicine,

Silesian Medical University, Batorego 15, 41-902 Bytom, Poland

\section{Introduction}

Disease of the venous system is a serious public health problem affecting all Western industrialized countries. The prevalence of lower-limb venous disease in the adult population is estimated to be at $40-50 \%$ for men and $50-55 \%$ for women, whereas visible varicose veins and chronic venous insufficiency are, respectively, present in $10-15 \%$ and $2-7 \%$ of the male population and $20-25 \%$ and $3-7 \%$ of the female population [1]. In the United States, an estimated $23 \%$ of adults have varicose veins, and $6 \%$ have more advanced chronic venous disease (CVD), including skin changes and healed or active venous ulcers [2]. However, CVDs are considered a cosmetic problem by some patients, although they may cause pain and discomfort, a loss of workdays, disability, and a deterioration of a healthy quality of life $[3,4]$. Therefore, CVDs make up a huge medical as well social problem. Duplex ultrasonography, the gold standard, may not show any pathologies in the venous system in the early stages of CVDs, and in some patients, venous disease may only be connected with venous microcirculation disorders. Hence, general practitioners and angiologists need an easy and nonivasive technique, which would allow them to diagnosis CVDs in their early stages.

The increase of internal organism temperature is usually treated like an indicator of different disease processes occurring the in organism. It is usually measured by contact thermometry.

However, skin temperature is also often the first symptom of disease in a living organism. Skin temperature can be easily evaluated with an infrared technique [5-11]. It is important to realize that particular skin temperature changes are associated with local blood flow in the most superficial skin layers, which makes thermovision extremely useful in medical diagnosis $[7,12]$. 
There are many different diseases that can manifest as changes not only to internal temperature but also to the skin surface temperature gradient. Mostly, they can be observed in spinal diseases-inflammatory states of vertebrates, skin ulceration, as well as different lesions like skin or breast cancers. In addition, some physical factors, such as the extremely low temperature used in cryotherapy, influence the internal temperature which trigger physiological reactions in response to cold, in addition to supporting basic therapy and facilitating kinesiotherapy, can lead to a significant increase of skin temperature contrast. Such an amplification of skin contrast is also used in thermovision diagnosis [5-14].

Another very interesting application of thermovision diagnosis seems to be venous embolisms of lower extremities, which are one of the biggest problems in medicine today. It is important that temperature changes and blood flow changes, especially in the most superficial skin layers, like the great and small saphenous veins, which can lead to gradient skin temperature changes that can be easily detected by thermovision [8].

As in various health specialties, angiology still needs fast, easy-to-use and nasive techniques for the early diagnosis of different veins diseases, due to their influence on our quality of life. On the other hand, we have to remember that venous diseases can lead to very serious health complications and consequences $[4,15,16]$.

There are many factors that can lead to venous diseases. However, one of the most important is vein hypertension $[15,16]$. It can be connected with an erroneous function of the vein's valves and a narrowing of the vessel's diameter. When several factors occur at the same time, it is possible to observe blood stasis and a filling up of a part of the blood circulation system. This leads to leucocyte entrapment (leucocytes can go out of the veins) and an inflammatory state in that area. The pressure and inflammatory state of the capillaries become more pervious to swelling. In some cases, in a very advanced state of disease, it is possible to see a widening of superficial veins that protrude along the skin. These are caused by varicose veins. Such changes are seen two times more often in women than men [4, 15-22]. Everything mentioned above can be seen in thermal imaging due to a differentiation in the temperature gradient on the tissue surface [5, 7-9].

Moreover, when the blood begins to be too sticky and coagulates too fast we must be concerned about the dangerous condition of vein thrombosis. It is a concern for old people, especially in their sixties or older, and obese people, and those who are suffering from varicose veins or those who are disabled and unable to move as a result of certain diseases. The long-lasting immobilization of the patient causes a work limitation in the muscles and consequently, a disorder in the circulation of blood.
When a rash occurs around wider veins, it may be superficial vein thrombophlebitis. There are many different symptoms which are connected with such changes. However, most of these symptoms lead to skin temperature changes especially when the diseases are connected with a superficial skin layer. This is the reason for using thermal imaging in such diseases. It should be also emphasized that skin changes that appear due to venous diseases are one of the most common health problems in industrialized countries. Thus, it is extremely important to find new diagnostic methods which are cheap and easy to use. Thermal imaging can show us temperature changes as a first symptom before the onset of tissue changes and big vessel's blood circulation problems. Thermovision is widely used in various applications in medicine, which include breast thermography, dermatological diseases, studies of inflammatory states, Raynaud phenomenon, foot ulceration among patients with diabetes mellitus, and lower back pain diseases where a temperature map of the body surface can give additional diagnostic information [5-14, 23, 24]. Notably, thermal measurements are very easy to perform and can provide some important information connected with blood supply and metabolism [7-9, 23, 24]. There are many other uses for thermal imaging for skin changes due to different physical factors, which were reported in previous articles [4-15].

It should be noted that thermal imaging can show from three to five times bigger areas characterized with a changed temperature gradient could be seen by a physician [25-28]. It is connected with the high sensitivity of thermovision cameras, patient metabolism, as well as circulation changes in capillaries (due to some diseases). As a result, thermal imaging may be useful in the study of skin or soft tissue diseases localized in the superficial skin layers.

Another important aspect of the skin that has an influence on temperature is the activity of the endothelium cells which excrete many active substances that are responsible for the vasoconstriction and vasodilatation of the vessels which play the main role in microcirculation.

There are a few main diagnosis techniques that are used in CVDs:

1. Duplex scanning allows for the evaluations of visibility, compressibility, and venous flow, including the measurement of the duration of reflux, and augmentation $[3,4]$.

2. Plethysmography (air or strain-gauge) is used for the noninvasive evaluation of calf muscle pump function, global venous reflux, and venous outflow obstruction [29].

3. Liquid crystal thermography, which is not common, is used and requires doing exercises-standing up on the toes and back on the heels 20 times, and then stick the 
liquid crystal plate to the tibia which allows one to see the inefficient lancination veins as hot spots [30].

4. Thermovision is very easy to perform and can give some important information connected to blood supply and metabolism changes on chosen skin areas. This technique may be effective, especially for the diagnosis of blood vessels close to the skin surface; they can be easily traced with thermal imaging that is sensitive to heat from blood vessels. However, it is not used as a standard technique [8].

Thermal imaging may give some possibilities to find not only visible pathological changes which can be diagnosed in duplex scanning but possibly also on areas of the skin where the pathological changes occur in capillaries, but are not visible in other diagnostic techniques.

Searching for quite simple and fast noninvasive techniques in medical diagnosis leads to finding new possibilities of using techniques like thermovision.

Metabolism and blood supply changes, and its intensity, manifests in body temperature changes, therefore the purpose of this work is to study skin temperature in chronic primary venous disease as well as try to find some relations between the known diagnostics factors, taken from duplex ultrasonography with temperature parameters obtained in thermal imaging. Such investigations may lead to finding some early symptoms of blood circulation disorders and may be useful in prophylaxis.

\section{Materials and methods}

The research group contained 14 patients ( 7 women and 7 male ages $60.3 \pm 11.0)$ suffering from primary venous disease.

The enrolment for the trial was performed in a group of patients with primary chronic disease stages $\mathrm{C} 2-\mathrm{C} 4$ who did not suffer from any other diseases, and in duplex scanning reflux duration $>500 \mathrm{~ms}$ in the great saphenous vein (GSV) the only deviation was found.

The term chronic venous disorder (CVD) is reserved for the full spectrum of venous abnormalities. The Guideline Committee recommends using the Classification of chronic venous disorders to document the clinical class, aetiology, anatomy, and pathophysiology (CEAP) of CVD. The classification is based on clinical signs of venous disease $(\mathrm{C})$, aetiology $(\mathrm{E})$, anatomy $(\mathrm{A})$, and the underlying pathophysiology (P). Clinical class includes the full spectrum of venous disorders, from no signs of visible venous disease $(\mathrm{C} 0)$ to telangiectasia or reticular veins $(\mathrm{C} 1)$, varicose veins $(\mathrm{C} 2)$, oedema $(\mathrm{C} 3)$, skin changes, such as pigmentation or eczema $(\mathrm{C} 4 \mathrm{a})$ or lipodermatosclerosis or atrophie blanche (C4b), and healed (C5) or active (C6) ulcer.
Aetiology can be congenital (Ec), primary (Ep), or secondary (Es). The main purpose of using the CEAP classification in patients with CVD is to distinguish primary venous disease from congenital varicosity and, most importantly, from secondary, post-thrombotic venous insufficiency. Evaluation and treatment of the three conditions are distinctly different.

Varicose veins of the lower limbs are dilated subcutaneous veins that are $\geq 3 \mathrm{~mm}$ in diameter measured in the upright position [3].

The investigations were carried out at the Chair and Clinic of Internal Diseases and Physical Medicine, Silesian Medical University in Bytom, Poland. All patients were examined by a physician. They were requested not to smoke, drink alcohol or hot drinks for at least $3 \mathrm{~h}$ before the thermovision studies. The thermal imaging of the skin surface was performed by using a Thermovision Camera A40M camera by Flir Systems, Sweden. All patients were diagnosed by duplex scanning (device Logic7) and the reflux duration as well as the range of reflux were determined. The emissivity was set in the range of $0.97-0.98$. The curves of the chosen regions of interests (ROI) were performed in a special room where the temperature was stabilized $\left(23 \pm 1{ }^{\circ} \mathrm{C}\right)$. The distance between the camera and the body was about $1.0-1.5 \mathrm{~m}$ (depending on the size of the limb). It was necessary to follow the standard protocol of infrared imaging in medicine [31].

There was also control group consisting of 10 healthy people of a similar age as a research group (5 women and 5 males in age $50.0 \pm 18.9$ and $22.2>\mathrm{BMI}>25$ ). All volunteers had done thermal images of their lower extremities in similar regions as the patients had.

All of the subjects signed a written consent for the diagnostics form, and they were previously informed about the plans and procedures.

The examination's protocol fulfils the ethical requirements of the 1975 Helsinki Declaration.

For the current study, the following parameters were taken into account:

- Mean venous reflux duration (VR), counted by duplex scan.

- Range of vein insufficiency (Range), counted by duplex scan.

- Mean temperature derived from isotherm area $\left(T_{\text {iso.area }}\right)$. Isotherm areas were marked using the mean temperature of the whole lower limb. The temperature above the mean limb temperature was taken as a temperature threshold used for the isotherm area in respective curves.

- Mean temperature of lower extremity $\left(T_{1 . e x t}\right)$, counted from curves. 
- Range of isotherm area (Thermal range), that describes the range of increase in temperature along the limb.

- Temperature difference $\left(\Delta T=T_{\text {iso.area }}-T_{1 \text {.ext }}\right)$

- Ratio of whole lower extremity area and isotherm area (Area\%).

Curves were analysed by the use of a ThermaCAM TM Researcher Pro 2.8 SR-3. Statistical analyses were done in Statistica 10 using $t$-tests, Wilcoxon's, Leven's and ANOVA tests. Differences with a $p<0.05$ were regarded as significant.

\section{Results and discussion}

Skin temperature is an indicator of the heat exchange between internal tissues and the environment in which the organism is located. This fact is illustrated in the curves of the representative objects in Figs. 1, 2, 3, 4, 5 and 6. Superficial venous diseases seem to be a very interesting and pertinent issue for thermal imaging.
It is easy to see the areas characterized with higher temperature, owing to changes as a result of diseaseinflammatory states connected with vein insufficiency. Consequently, the diagnostic values of the thermal imaging of venous embolisms of the lower limbs veins were analysed.

The curve in Fig. 1a reveals a prolonged area on the left thigh ending below the knee. It may be correlated with the great saphena vein insufficiency and the inflammatory state of soft tissue around it, which occurred in this patient. The contralateral thigh seems to be bigger because of swelling and the mean temperature of the left thigh is $0.2{ }^{\circ} \mathrm{C}$ lower than the right.

Figure 1b, c present longer areas characterized with a higher temperature for two patients suffering from vena saphena magna insufficiency. However, it is seen that the areas revealed on the skin are longer and more focused in the tracks of the veins. Figure $1 \mathrm{~b}$ shows an elongated area passing through the left limb from the thigh to half of tibia. Most likely, it is connected with a bigger range of vein insufficiency.
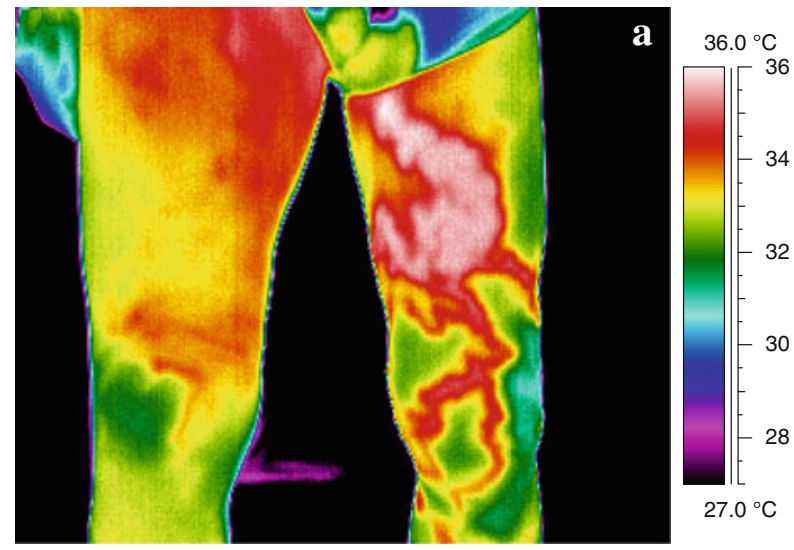

\begin{tabular}{cc}
\hline Area & Temperature ${ }^{\circ} \mathrm{C}$ \\
\hline Left thigh : & 33.7 \\
$T_{\text {mean }}$ &
\end{tabular}

Right thigh :

$T_{\text {mean }}$
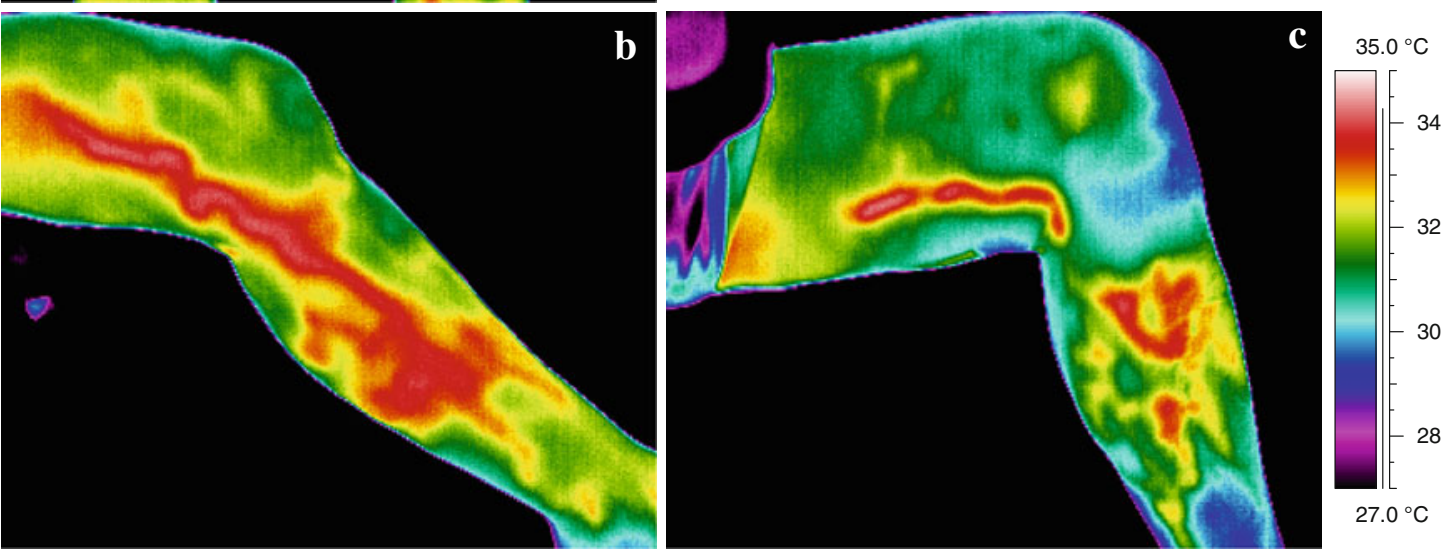

Fig. 1 The curves of the inside limb parts suffering from an insufficiency in the great saphenous vein of the lower left limb. a presents a patient with great saphenous vein insufficiency and an inflammatory state in the surrounding soft tissues. b, c present curves for two patients suffering from great saphenous vein insufficiency. Temperature parameters collected in the table show differences in mean temperatures between the left and right thighs 


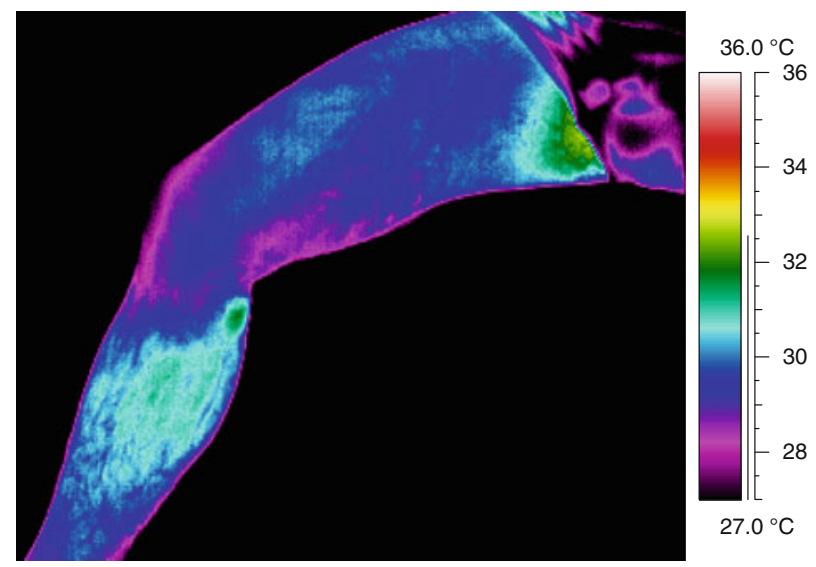

\begin{tabular}{cc}
\hline Area & Temperature ${ }^{\circ} \mathrm{C}$ \\
\hline
\end{tabular}

Right lower

limb : $T_{\text {mean }}$
29.8

Fig. 2 Curves of inside limb parts representative for healthy group

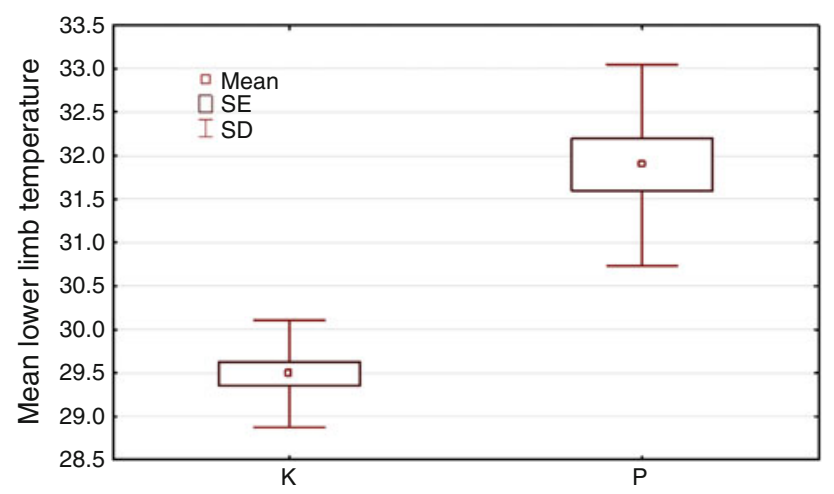

Fig. 3 The mean temperature of the lower extremities shows the differences obtained between healthy patients and those with vein insufficiency. Statistical values used: mean, SE and SD, mean value of temperature, standard error, and standard deviation

All curves of the thighs show elongated areas characterizing tracks of the GSV with a higher temperature than can be connected with pathological changes in the veins (Fig. 1). The enhancement of temperatures in some areas of the thighs seems to be associated with blood stasis because of a malfunction in the vein's valves. Such processes may lead to vein deformation and inflammatory states which manifest on the skin surface as areas with a higher temperature, unlike the healthy lower limbs (Fig. 1a). It appears that the differences in the temperature gradient can be easy correlated with the medical diagnosis.

The main processes that influence blood flow and the health of blood vessels are leucocyte entrapment, hyperviscosity of blood, and fibrin cuff. A number of blood morphotic elements have impact on the blood flow regulation in capillaries. The importance lies not only in the viscosity but also in the interaction with endothelium cells. It is known that leucocytosis cause an increase in resistance and in blood pressure, which is also connected with the flow of monocytes and granulocytes inside the capillaries. In addition, the erythrocytes are smaller than white blood cells and move faster, which can lead to a phenomenon called a leucocytes-erythrocytes train which causes an aggregation of erythrocytes that push the white blood cells towards the vessel walls. Such a situation leads to a rolling of the leucocytes process and their adhesion to endothelium cells, and an accumulation of leucocytes in what is known as leucocytes entrapment. Moreover, due to structural and functional changes in the endothelium as well in the leucocytes, it can lead to a migration of leucocytes through the blood vessel walls [4, 16]. All of the processes mentioned above take place in small vessels so that they cannot be visible in the duplex scan, but thermovision can show differences in temperature because of decreased blood flow, an increase of friction, as well as viscosity because of their influence on heat production. In addition, due to the release of cytotoxic substances and the changes in blood cells, the vessel walls can be damaged which enables the increase of permeability and inflammation. One can easily observe the results of these processes because they are able to show the gradient temperature changes-mostly the increase of the localized temperature connected with route of the veins.

One of the stages of venous diseases can produce a swelling of soft tissue in the limbs and cause an increase of blood pressure, narrowing of capillaries, and decrease of blood flow, and lead to ischaemia and oxygen deficiency in the tissues. This also can be manifested by changes of the temperature gradient-mostly a decrease of temperature in the soft tissues due to slow thermoregulation. Similar effects may be observed because of damage of the endothelium, and it leads to swelling (Fig. 1a, right limb). Fluid contains protein such as fibrinogen which leads to building fibrinogens edging around the capillaries and a handicap of gas exchange and anoxia [4, 16]. All of these processes 
Fig. 4 Curves of representative patients suffering from great saphenous vein insufficiency and respective images, with marked isotherm areas. The temperature above the mean limb temperature was taken as a temperature threshold used for the isotherm area in respective curves
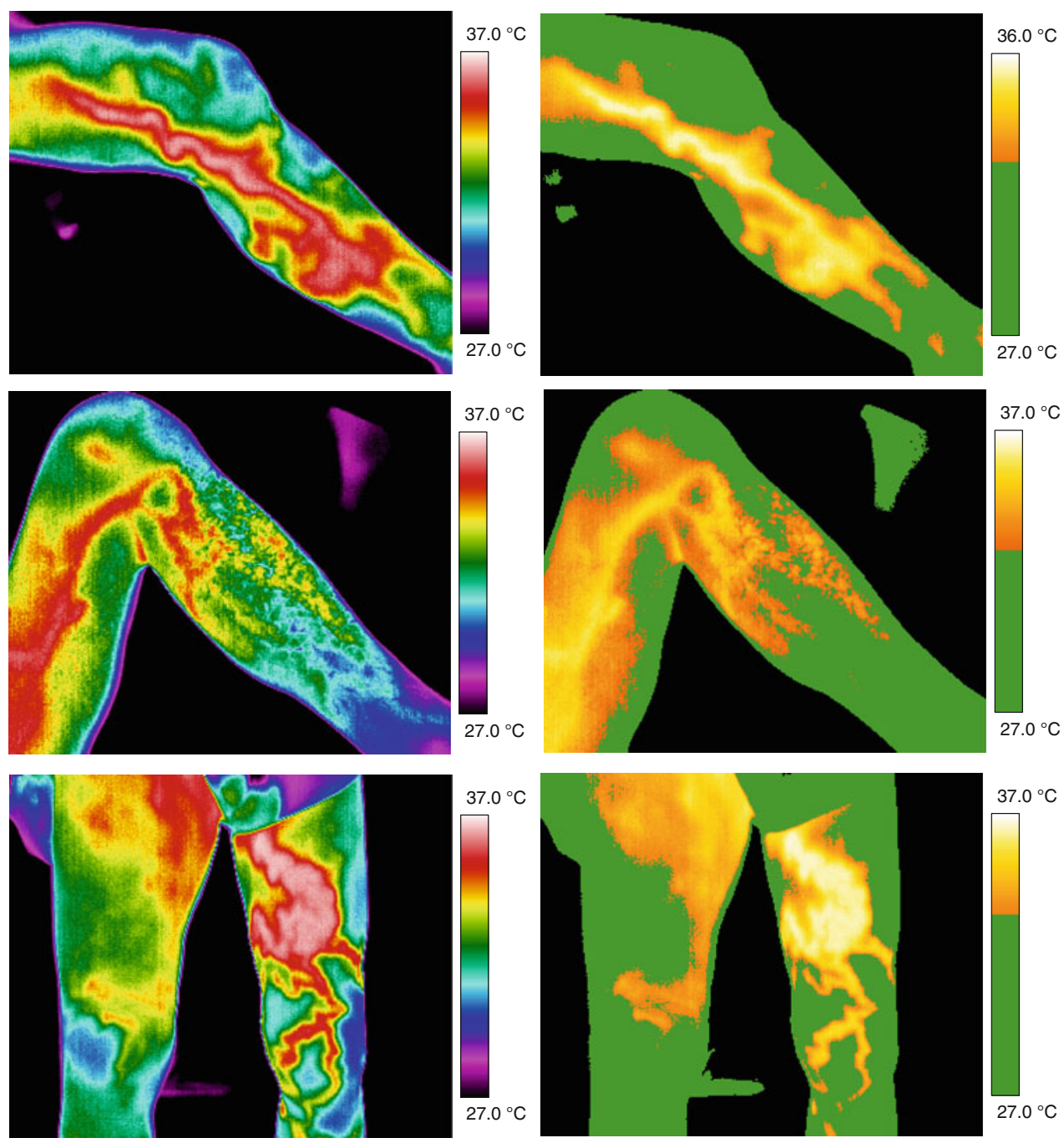

induce changes in the metabolism of the soft tissues and the skin surface, which are easily seen in the curves (Fig. 1).

The next process which can be observed during the development of vein insufficiency is the migration of leucocytes towards the main skin which leads to a development of pathological processes and, as a result, to trophic ulceration, which was reported in previous papers $[8,9]$.

Such skin temperature effects are not visible in healthy limbs. Curves do not show large temperature gradient changes along the tracks of the superficial veins (Fig. 2), and the skin temperature is much lower than for the affected patients (Fig. 3).

To enable us see the variation between controls better, all temperature results were collected in the Table 1 .

Thus, we can emphasize that skin temperature changes connected with problematic blood circulation can be easily seen in thermovision especially on the skin surface or superficial skin layers in the vicinity of blood vessels.

For gaining a deeper insight into the problem, a statistical analysis was done. It compared the differences between the mean temperature of the lower extremities for patients with CVDs, vein insufficiency and the healthy ones (Fig. 3).

Problems with blood microcirculation and vein hypertension are the most dominant reasons for the origin of typical symptoms of chronic vein insufficiency. It can lead to swelling in soft tissues and skin temperature changes. It appears that such a process may be observed in Fig. 4c (right lower limb). The isotherm area is larger on the left limb than on the right, and the mean temperature of the left limb surface is higher than that of the right (Fig. 1a). It is due to limited blood circulation in the capillaries of the left limb and is associated with slower metabolism on the skin 


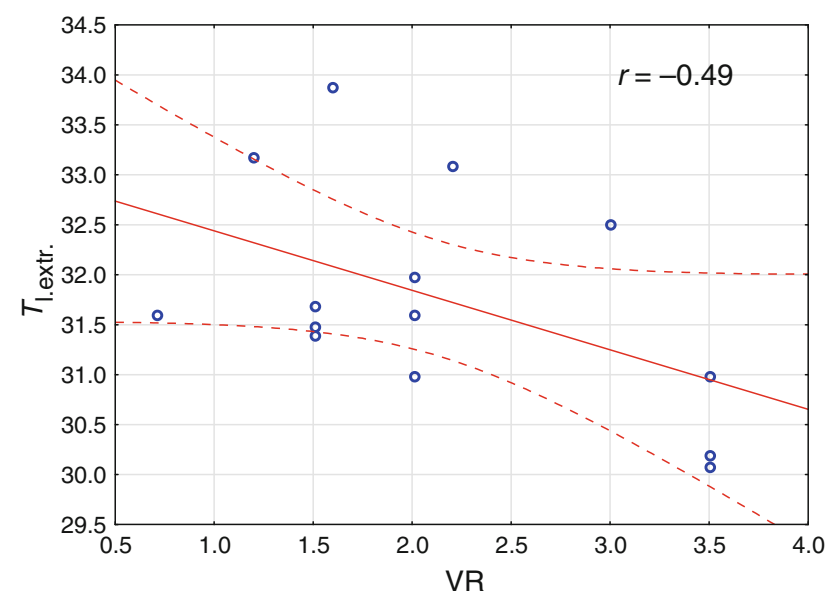

Fig. 5 Correlation between mean Venous Reflux Duration and Lower Limb mean temperature $\left(T_{\text {l.extr }}\right)$ obtained for all studied patients, where $r$ is the correlation coefficient

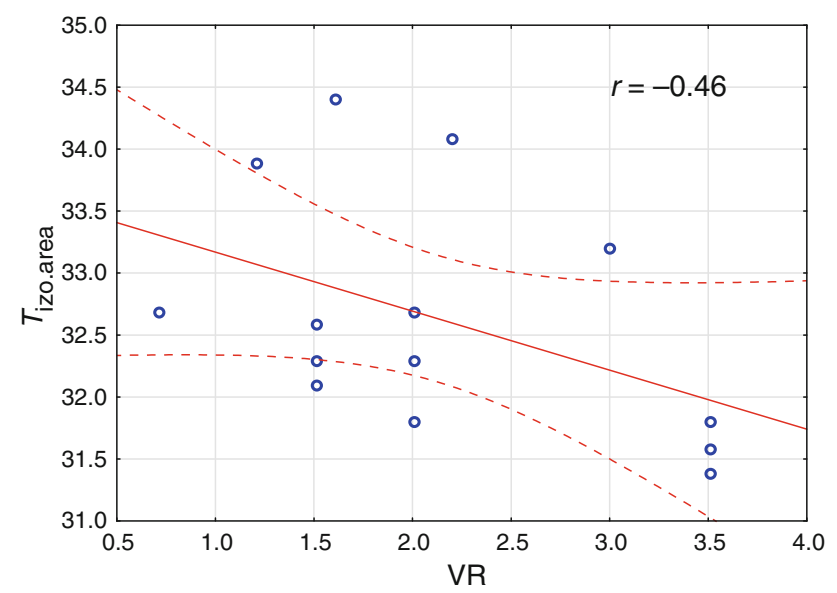

Fig. 6 Correlation between mean Venous Reflux Duration and mean temperature of isotherm area $\left(T_{\text {iso.area }}\right)$ obtained for all studied patients, where $r$ is the correlation coefficient

surface. The next stage of the disease is observed when a stagnation of blood in the veins leads to an opening of the connections between arteries and veins, which is protective activity, and leads to a decrease of hydrostatic pressure in the capillaries, as represented in Figs. 4a, b. However, the next stage of the disease leads to the exclusion of a whole part of the capillary microcirculation system. Excluded areas of microcirculation cause ischaemia and anoxia in soft tissues and venous stasis and a filtration of blood and fluid to extracellular space.

Moreover, a dysfunction of fibrinolysis leads first to embolism inside the capillaries and next, to hypoxia and tissue necrosis. Such processes were discussed in other papers concerned with trophic ulcerations treated in hyperbaric chambers $[8,9]$.
Table 1 The temperature parameters: mean temperature of lower extremity $\left(T_{1 . \text { ext }}\right)$ of control group, mean temperature of control group $\left(T_{\text {l.ext.control }}\right)$ and standard deviation obtained for control group

\begin{tabular}{|c|c|c|}
\hline Patient & Lower extremity & $\begin{array}{l}\text { Mean temperature, } \\
T_{\text {l.ext.control }} /{ }^{\circ} \mathrm{C}\end{array}$ \\
\hline \multirow[t]{2}{*}{1} & Right & 28.4 \\
\hline & Left & 28.6 \\
\hline \multirow[t]{2}{*}{2} & Right & 29.3 \\
\hline & Left & 29.3 \\
\hline \multirow[t]{2}{*}{3} & Right & 29.7 \\
\hline & Left & 29.8 \\
\hline \multirow[t]{2}{*}{4} & Right & 29.2 \\
\hline & Left & 29.3 \\
\hline \multirow[t]{2}{*}{5} & Right & 28.7 \\
\hline & Left & 28.9 \\
\hline \multirow[t]{2}{*}{6} & Right & 30.6 \\
\hline & Left & 30.9 \\
\hline \multirow[t]{2}{*}{7} & Right & 29.8 \\
\hline & Left & 29.9 \\
\hline \multirow[t]{2}{*}{8} & Right & 29.4 \\
\hline & Left & 29.3 \\
\hline \multirow[t]{2}{*}{9} & Right & 29.5 \\
\hline & Left & 29.4 \\
\hline \multirow[t]{2}{*}{10} & Right & 29.8 \\
\hline & Left & 30 \\
\hline \multicolumn{2}{|c|}{$T_{\text {mean }} /{ }^{\circ} \mathrm{C}$} & 29.5 \\
\hline \multicolumn{2}{|c|}{ Standard deviation (SD) } & 0.6 \\
\hline
\end{tabular}

The very important thing is that all of the processes mentioned above relate to capillaries and microcirculation in the skin and superficial skin layer. That is why it may cause the tissue temperature gradient changes and reflect on skin as an abnormal temperature gradient. This problem seems to show a correlation between the temperature parameters obtained from the curves and the duplex scanning parameters, like the mean reflux duration and the range of vein insufficiency, which is the aim of the next part of this study.

All parameters used in the statistical analysis and correlation are displayed in Table 2.

To find out if there are any dependencies between the duplex parameters (VR and Range) and the temperature parameters (mean temperature derived from isotherm area ( $\left.T_{\text {iso.area }}\right)$, mean temperature of lower extremity $\left(T_{\text {l.ext }}\right)$, range of isotherm area (Thermal range), temperature difference $\left(\Delta T=T_{\text {iso.area }}-T_{\text {l.ext }}\right)$, ratio of whole lower extremity area and isotherm area (Area\%)), correlations were performed, and a few selected results are presented in Figs. 5, 6, 7 and 8 .

It can be clearly seen from Figs. 5 and 6 that there is a medium negative correlation between the mean Venous 
Table 2 The temperature parameters (mean temperature derived from isotherm area $\left(T_{\text {iso.area }}\right)$, mean temperature of lower extremity $\left(T_{1 . e x t}\right)$, range of isotherm area (Thermal range), temperature difference $\left(\Delta T=T_{\text {iso.area }}-T_{\text {l.ext }}\right)$, ratio of whole lower extremity area and isotherm area (Area\%), and duplex parameters (mean venous reflux duration (VR), range of vein insufficiency (Range) obtained from the whole group of patients

\begin{tabular}{|c|c|c|c|c|c|c|c|}
\hline Patient number & $\mathrm{VR} / \mathrm{s}$ & Range & $T_{\text {izo.area }}$ & $T_{1 \_ \text {extr }}$ & Thermal range & $\Delta T$ & $\begin{array}{l}\text { (Izo.Area)/ } \\
\text { (1_extr.Area)/\% }\end{array}$ \\
\hline 1 & 2 & 2 & 31.8 & 31 & 0.73 & 0.8 & 0.46 \\
\hline 2 & 3.5 & 4 & 31.8 & 31 & 0.76 & 0.8 & 0.42 \\
\hline 3 & 3.5 & 4 & 31.6 & 30.1 & 1 & 1.5 & 0.3 \\
\hline 4 & 3.5 & 4 & 31.4 & 30.2 & 0.9 & 1.2 & 0.4 \\
\hline 5 & 3 & 3 & 33.2 & 32.5 & 0.8 & 0.7 & 0.41 \\
\hline 6 & 1.5 & 3 & 32.1 & 31.4 & 0.67 & 0.7 & 0.37 \\
\hline 7 & 2 & 4 & 32.7 & 32 & 0.81 & 0.7 & 0.46 \\
\hline 8 & 0.7 & 3 & 32.7 & 31.6 & 0.88 & 1.1 & 0.49 \\
\hline 9 & 1.5 & 2 & 32.6 & 31.5 & 0.75 & 1.1 & 0.42 \\
\hline 10 & 1.2 & 4 & 33.9 & 33.2 & 0.83 & 0.7 & 0.46 \\
\hline 11 & 2.2 & 4 & 34.1 & 33.1 & 0.92 & 1 & 0.41 \\
\hline 12 & 2 & 4 & 32.3 & 31.6 & 0.74 & 0.7 & 0.34 \\
\hline 13 & 1.5 & 3 & 32.3 & 31.7 & 0.77 & 0.6 & 0.42 \\
\hline 14 & 1.6 & 4 & 34.4 & 33.9 & 0.78 & 0.5 & 0.38 \\
\hline
\end{tabular}

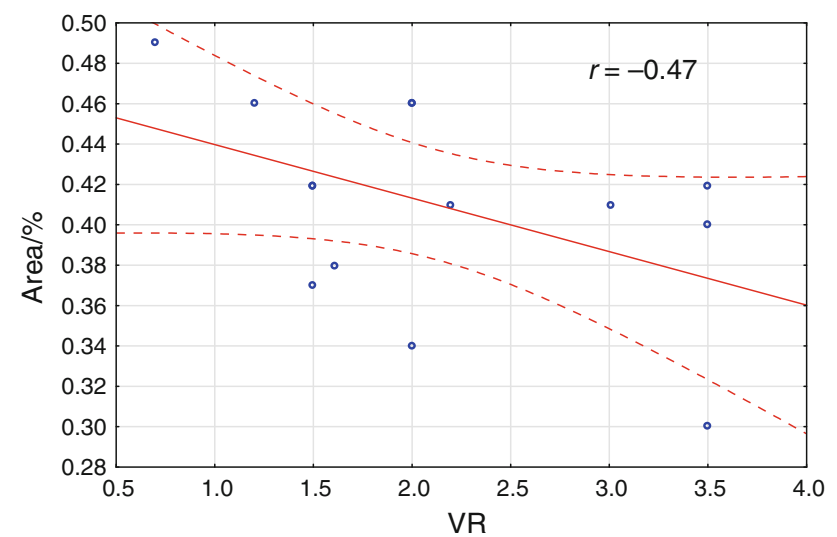

Fig. 7 Correlation between mean Venous Reflux Duration and ratio of whole lower extremity area and isotherm area (Area\%) obtained from all patients, where $r$ is correlation coefficient

Reflux Duration and the Lower Limb mean temperature as well as the mean Venous Reflux Duration and the mean temperature of the isotherm area. It shows a very interesting result. High mean venous reflux duration can be connected with a quite low blood circulation and a dysfunction of the thermoregulation in the soft tissues of the lower extremities. This is explainable by noticing that when reflux is quite low, the inflammatory state that occurs around the vein track in the limb is also smaller due to better blood circulation, compared with cases of higher values in the reflux parameter. In these cases, the isotherm area is larger than in the cases of high reflux, and the mean limb temperature is higher. It may be caused by a better

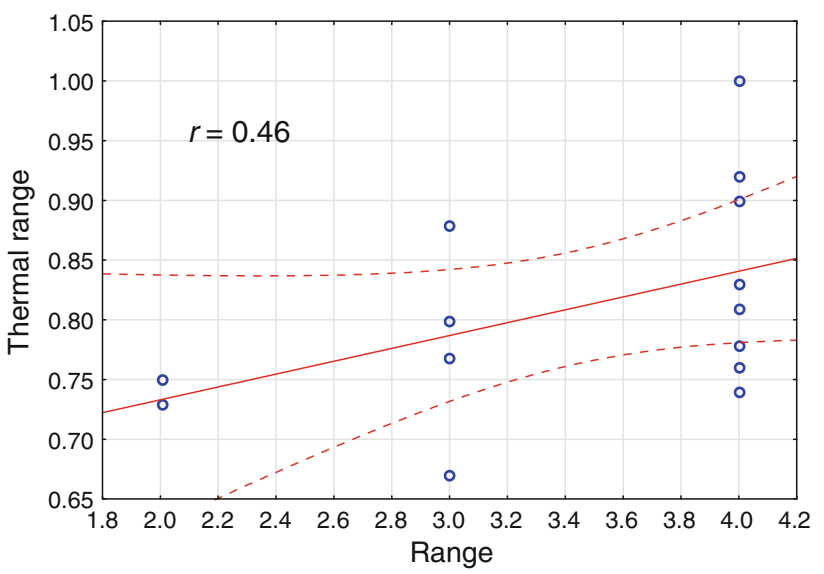

Fig. 8 Correlation between thermal range defined as range of isotherm area (described as range of increase temperature along the limb) and range of great saphenous vein insufficiency obtained for all patients, where $r$ is correlation coefficient

unified skin temperature because of a small inflammatory state and small tissue parts excluded from the whole part of the microcirculation capillaries system (Fig. 7). On the other hand, excluded parts of microcirculation causes ischaemia and anoxia of soft tissues and bigger venous stasis which may be the reason for a lower blood supply and lead to colder lower extremities. The patients may sense this by touching their limb surface with their hands. The next stage of the disease leads to structural and functional changes in the endothelium and causes a migration of leucocytes through the blood vessel walls. It can be manifested in a wider skin temperature gradient and a 
ramification of the vessels due to a big inflammatory process.

It was also interesting to find the answer to the question of if it is possible to fix the range of vein insufficiency (range), counted by duplex scanning just by means of the thermal imaging. The correlation between the thermal range, defined as the range of increased temperature (isotherm) along the limb, and the range of vein insufficiency, is presented in Fig. 8.

In this case, a medium correlation was obtained ( $r=0.46)$. It means that there is some correlation between the thermal range defined as the range of isotherm area (described as a range of increased temperature along the limb) and the range of GSV insufficiency counted by a duplex scan. It may be possible to optimize this thermal method of vein insufficiency estimation, but we need a bigger study group and an improved as well as automatic algorithm for solving the range. It should be a faster, cheaper, and easier method to use, at least in screening or for first contact with a physician, just to determine if there are some temperature gradient changes which might be connected with venous/circulation diseases.

The use of thermal imaging as a diagnostic or screening technique in the case of superficial venous diseases is promising. It may give us thermal images which are correlated with a state of the veins and small vessel blood circulation. We can also see that changes of the limb temperature gradient during the development of disease stays in good correlation with the physiological processes occurring in soft tissues and capillaries. In addition, many details can be seen which could not be seen in duplex scanning. Thermal imaging is used as a technique that can show the metabolism and functional changes manifested as changes in skin temperature. However, every patient should be taken into consideration individually. It is very important to evaluate the standard protocol for using thermovision for vein insufficiency diseases. Moreover, it should be noted that the research group in this study is small, and so the conclusion must be drawn very cautiously and thus, we need further confirmation based on the study with a larger group of subjects.

\section{Conclusions}

Thermal imaging as a completely noninvasive technique can be helpful in the diagnosis of superficial vein insufficiency.

It may be possible to use thermovision as a helpful imaging technique which can be used as a screening method alone or together with ultrasound diagnosis because of some correlation between these two method's parameters; for confirming this, however, further studies with a larger number of patients in the study group will be needed.
Acknowledgements The authors would like to thank the patient subjects who volunteered for participation in the study.

Open Access This article is distributed under the terms of the Creative Commons Attribution License which permits any use, distribution, and reproduction in any medium, provided the original author(s) and the source are credited.

\section{References}

1. Van den Oever R, Hepp B, Debbaut B, Simon I. Socio-economic impact of chronic venous insufficiency. An underestimated public health problem. Int Angiol. 1998;17:161-7.

2. Kaplan RM, Criqui MH, Denenberg JO, Bergan J, Fronek A. Quality of life in patients with chronic venous disease: San Diego population study. J Vasc Surg. 2003;37:1047-53.

3. Gloviczki P, Comerota AJ, Dalsing MC, Eklof BG, Gillespie DL. The care of patients with varicose veins and associated chronic venous diseases: clinical practice guidelines of the Society for Vascular Surgery and the American Venous Forum. J Vasc Surg. 2011;53(5): 1-48.

4. Sieroń Aleksander, Cierpka Lech, Rybak Zbigniew, Stanek Agata. Angiology textbook. Bielsko Biała: Alfa Medica Press; 2009.

5. Cholewka A, Drzazga Z, Sieroń A, Stanek A. Thermovision diagnostics in chosen spine diseases treated by whole-body cryotherapy. J Therm Anal Calorim. 2010;102:113-9.

6. Cholewka A. Diagnostic meaning of thermographic analysis of whole body cooling in cryotherapy. In: Skrzek A, Podbielska H, editors. Applications of low temperature in medicine. Polna: OWPW; 2012 (in Polish).

7. Cholewka A, Stanek A, Kwiatek S, Sieroń S, Drzazga Z. Does the temperature gradient correlate with photodynamic diagnosis parameter NCV? Photodiagn Photodyn Ther. 2013;10:33-8.

8. Cholewka A, Drzazga Z, Sieroń A, Stanek A, Knefel G et all (2010). In: (eds.) Drzazga Z, Ślosarek K Some applications of thermal imaging in medicine in Some aspects of medical physics-in vivo and in vitro studies. Olsztyn: Hard Publishing Company Olsztyn 2010: 51-8.

9. Cholewka A, Knefel G, Stanek A, Kawecki M, Nowak M, et al. Thermal imaging and TC oximetry measurements of hyperbaric oxygen therapy (HBO) effects on trophic ulceration of the crura. J Therm Anal Calorim. 2012;108:25-31.

10. A. Cholewka, Z. Drzazga, G. Knefel, M. Kawecki, M. Nowak (2010) Thermal imaging in hyperbaric oxygen therapy (HBO), Inżynieria Biomedyczna.

11. Ammer K. Temperature effects of thermotherapy determined by infrared measurements. Physica Media. 2004;20(Suppl I):64-6.

12. Cholewka A, Drzazga Z, Michnik A, Sieroń A, Wiśniowska B. Temperature effects of whole cryotherapy determined by thermography. Thermol Intern. 2004;14:57-63.

13. Mercer JB, Ring EFJ. Fever screening and infrared thermal imaging: concerns and guidelines. Thermol Int. 2009;19:67-9.

14. Nowakowski A. Thermography progresses-medical applications. Gdańsk: Wydawnictwo Gdańskie; 2001.

15. Wilkinson LS, Bunker C, Edwards JC, Scurr JH, Smith PD. Leukocytes: their role in the etiopathogenesis of skin damage in venous disease. J Vasc Surg. 1993;17(4):669-75.

16. Hess CT. Identifying and managing venous dermatitis. Adv Skin Wound Care. 2005;18(5 Pt 1):242-3.

17. Bollinger A, Isenring G, Franzeck UK. Lymphatic microangiopathy: a complication of severe chronic venous incompetence (CVI). Lymphology. 1982;15(2):60-5.

18. Bollinger A, Leu AJ, Hoffmann U, Franzeck UK. Microvascular changes in venous disease: an update. Angiology. 1997;48(1): $27-32$. 
19. Bradbury AW, Murie JA, Ruckley CV. Role of leucocyte in the pathogenesis of vascular disease. Br J Surg. 1993;80(12): 1503-12.

20. Einspahr JG, Thomas TL, Saboda K, Nickolof BJ, Warneke J, et al. Expression of vascular endothelial growth factor in early cutaneous melanocytic lesion progression. Cancer. 2007;110(11):2519-27.

21. Shor NA, Pronin IV. Local signs of the reperfusion syndrome in obliterating atherosclerosis of the lower extremity arteries and their treatment. Klin Khir. 2006;1:34-7.

22. Sukovatykh BS, Kniazev VV. The mechanisms of critical impairments of microcirculation in patients with chronic ischemia of lower extremities. Vestn Khir Im I I Grek. 2007;166(4):20-4.

23. Ammer K. Temperature effects of thermotherapy determined by infrared measurements. Phys Med. 2004;20(Suppl. I):64-6.

24. Ring EFJ. Provocation tests in thermal imaging, Abs. Thermology Int. 11, 2, 2001.

25. de Weck AL, Gluck U, Derer T. Thermographic analysis of allergic reactions in the skin. $\mathrm{J}$ Allergy Clin Immunol News. 1990;2:7-10.

26. de Weck AL, Gluck U, Bahre M. Investigation of the anti-allergic activity of azelastine on the immediate and late phase reactions to allergens and histamine using telethermography. Clin Exp Allergy. 2000;30:283-7.
27. Siebennaar F, Degener F, Zuberbier T, Martus P, Maurer M. High-dose desloratadine decreases wheel volume and improves cold provocation thresholds compared with standard-dose treatment in patients with acquired cold urticaria: a randomized, placebo-controlled, crossover study. J Allergy Clin Immunol. 2009;123:672-9.

28. Tomasz Rok, The use of thermovision in diagnostics and therapy, Doctor thesis, 2012, Jagiellonian Univeristy Kraków. (spr).

29. Nicolaides AN, Cardiovascular Disease Educational and Research Trust, European Society of Vascular Surgery, the International Angiology Scientific Activity Congress Organization, International Union of Angiology, Union Internationale de Phlebologie at the Abbaye des Vaux de Cernay. Investigation of chronic venous insufficiency: a consensus statement (France, March 5-9, 1997). Circulation. 2000;129:E126-63.

30. Cameron EW, Sachdev D, Gishen P, Martin JF. Liquid crystal thermography as a screening test for deep vein thrombosis in patients with cerebral infarction. Eur J Clin Invest. 1991;21(5):548-50.

31. Ring EFJ, Ammer K. The technique of infrared imaging in medicine. Thermol Intern. 2000;10:7-14. 